\title{
The Seventeenth International Symposium on Relations Between Homogeneous and Heterogeneous Catalysis Utrecht July 12-15 2015
}

\author{
Bert M. Weckhuysen ${ }^{1} \cdot$ Pieter C. A. Bruijnincx ${ }^{1} \cdot$ Monique F. A. Lamers $^{1}$ • \\ Robertus J. M. Klein Gebbink ${ }^{2}$
}

Published online: 25 August 2016

(c) Springer Science+Business Media New York 2016

The series of International Symposia on Relations between Homogeneous and Heterogeneous Catalysis (ISHHC) found its origin in 1974 in Brussels (Belgium). It was immediately considered a very successful and inspiring scientific gathering with more than 230 participants from 24 different countries. Interestingly, the title of the first symposium was slightly different, namely "International Symposium on the Relations between Heterogeneous and Homogeneous Catalytic Phenomena". As formulated in the editorial of the Proceedings by Professor Bernard Delmon (Université catholique de Louvain, Belgium), the purpose of the symposium was to bring scientists together to discuss "the common features of homogeneous and heterogeneous catalytic phenomena and similitudes of active species or elementary steps in both systems with explicitly stated topics on (a) heterogeneous catalytic phenomena, which exhibit some fundamental aspects of homogeneous catalysis, (b) heterogenization of coordination complexes and (c) catalysis by soluble metal aggregates". The scientific father of the first ISHHC symposium was Professor José Fripiat, also at that time at the Université catholique de Louvain, who felt that a scientific meeting on these topics would give new direction and inspiration to scientists working in the field of catalysis. Till today his scientific heritage remains at the core of this important

Bert M. Weckhuysen

b.m.weckhuysen@uu.nl

1 Inorganic Chemistry and Catalysis, Debye Institute for Nanomaterials Science, Utrecht University, Universiteitsweg 99, 3584 CG Utrecht, The Netherlands

2 Organic Chemistry and Catalysis, Debye Institute for Nanomaterials Science, Utrecht University, Universiteitsweg 99, 3584 CG Utrecht, The Netherlands
Symposia series, which had its most recent 17 th edition in 2015 in Utrecht (Fig. 1).

The Utrecht symposium attracted around 450 participants from more than 40 different countries, illustrating the truly international nature of the event. Thirty percent of the participants were $\mathrm{PhD}$ students. Building on the rich tradition of the series (see Table 1 for a historical overview of past locations), ISHHC17 aimed to link the different fields of catalysis, namely homogeneous and heterogeneous catalysis, with the goal to understand catalytic phenomena at the molecular level and by doing so uncover new aspects as well as similar concepts shared by both catalytic approaches. For this, a scientific program was assembled with world leading plenary and keynote speakers, 67 regular oral contributions and over 200 poster presentations. The scientific program had been divided over four topical sessions, namely "Advanced Tools for Catalysis", which was co-chaired by Suzanne Blum (University of California, Irvine, USA), Maarten Roeffaers (KU Leuven, Leuven, Belgium) and Bert Weckhuysen; "Complexity and Cooperativity in Catalysis", co-chaired by Bas de Bruin (University of Amsterdam, Amsterdam, The Netherlands), Masaya Sawamura (Hokkaido University, Sapporo, Japan) and Bert Klein Gebbink; "Hybrid Approaches to Catalysis", co-chaired by Kuiling Ding (Chinese Academy of Sciences, Shanghai, China), Justin Notestein (Northwestern University, Evanston, USA) and Pieter Bruijnincx; and Reaction and Separation Technology", co-chaired by Eelco Vogt (Albemarle Catalysts, Amsterdam, The Netherlands), Christopher Hardacre (Queen's University Belfast, Belfast, United Kingdom) and Walter Leitner (RWTH Aachen, Aachen, Germany). Next to these four dedicated sessions, there was a general session as well as two poster sessions. This, together with a social program, allowed for ample opportunities for scientific discussion. 


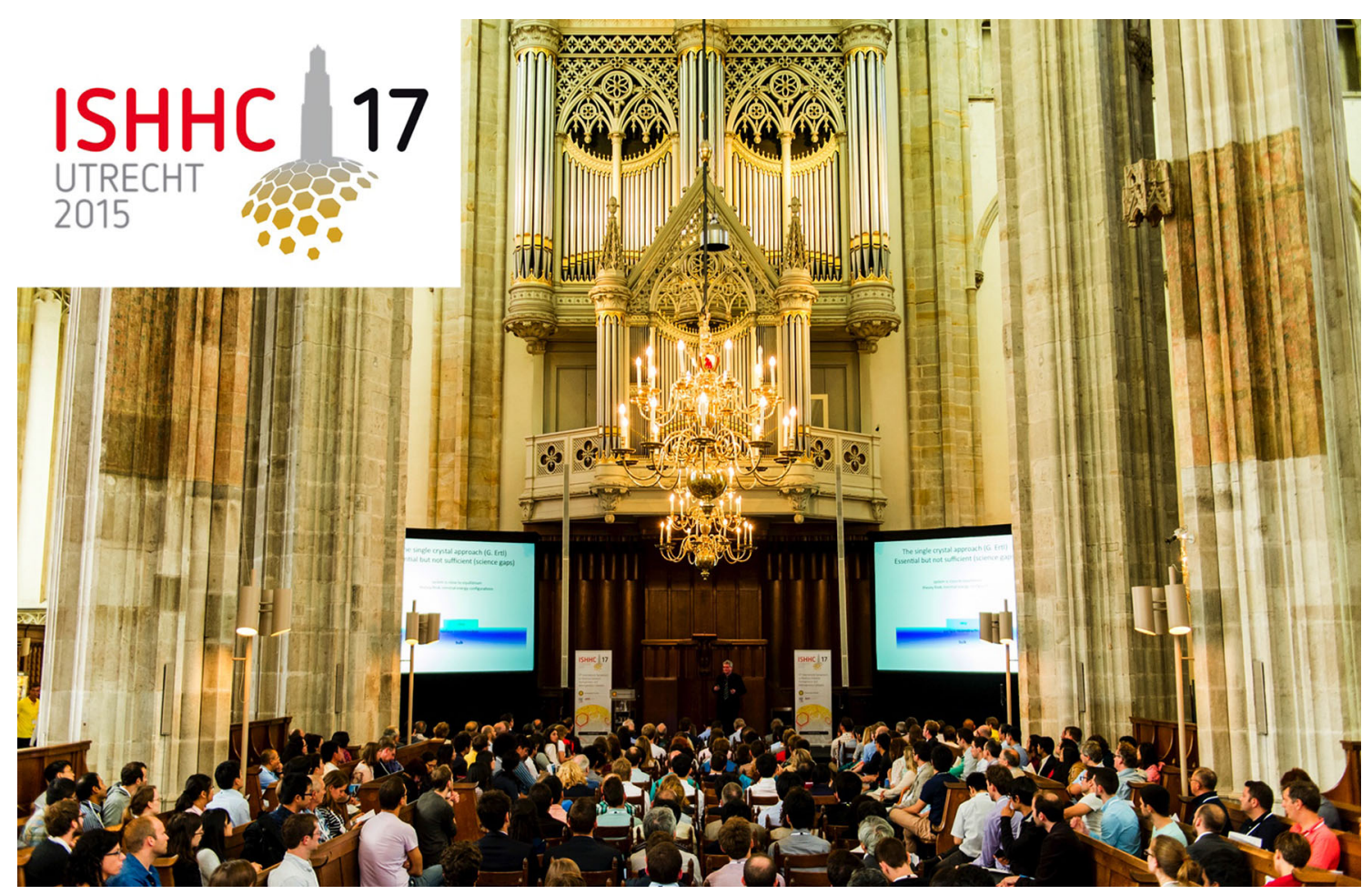

Fig. 1 Opening lecture in the Dom Church in the city center of Utrecht by Professor Robert Schlögl (Fritz Haber Institute of the Max Planck Society, Berlin, Germany) during the 17th International

Table 1 Historical overview of the years and places of the series of International Symposia on Relations between Homogeneous and Heterogeneous Catalysis

\begin{tabular}{llll}
\hline 1974 & Brussels & 1999 & Southampton \\
1977 & Lyon & 2001 & Lyon \\
1981 & Groningen & 2003 & Evanston \\
1983 & Asilomar & 2005 & Florence \\
1986 & Novosibirsk & 2007 & Berkeley \\
1989 & Pisa & 2009 & Stockholm \\
1992 & Tokyo & 2011 & Berlin \\
1995 & Balatonfured & 2013 & Sapporo \\
& & 2015 & Utrecht \\
\hline
\end{tabular}

This themed Topics in Catalysis issue comprises a selection of 13 research articles, concerned with results presented at ISHHC17. They clearly illustrate the breadth and scope of contemporary research in the fields of heterogeneous and homogeneous catalysis, and some of them specifically address the inter-relationships between both fields of catalysis research. They cover different types of active sites: metal, redox, acid as well as base sites. Also of importance here is to mention the advances made in both
Symposium on Relations between Homogeneous and Heterogeneous Catalysis (July 12-15 2015) Photo: Marco De Swart

our experimental and theoretical understanding of the complexity of catalyst systems. This includes studies on the dynamics of active sites when catalyst materials are synthesized, function and deactivate. We truly hope you will enjoy these scientific contributions.

We wish to express our gratitude to all the sponsors and exhibitors, 28 in total, who provided generous financial support and contributed greatly to the success of the conference. Their support was used specifically to reduce the registration fees for $\mathrm{PhD}$ students. Also a special word of gratitude should go to Utrecht University, who via its Strategic Theme Sustainability, contributed substantially to this symposium by financing the conference secretariat. We also acknowledge the help and support from the National and International Advisory Boards and the Industrial Advisory Board, as well as the many $\mathrm{PhD}$ candidates and postdoctoral fellows who have helped with the practical aspects of the organization of ISHHC17. Having enjoyed the Utrecht meeting as organizers and participants ourselves, we very much look forward to the next edition of the meeting, which will take place in Sydney in 2018 and will be organized by Professor Thomas Maschmeyer and his team. 